\title{
BMJ Open Incidence and prognostic impact of post discharge bleeding post acute coronary syndrome within an outpatient setting: a systematic review
}

\author{
Nafiu Ismail, ${ }^{1}$ Kelvin P Jordan, ${ }^{1}$ Sunil Rao, ${ }^{2}$ Tim Kinnaird, ${ }^{3}$ Jessica Potts, ${ }^{1}$ \\ Umesh T Kadam, ${ }^{4}$ Mamas A Mamas ${ }^{1}$
}

To cite: Ismail N, Jordan KP, Rao S, et al. Incidence and prognostic impact of post discharge bleeding post acute coronary syndrome within an outpatient setting: a systematic review. BMJ Open 2019;9:e023337. doi:10.1136/ bmjopen-2018-023337

- Prepublication history and additional material for this paper are available online. To view these files, please visit the journal online (http://dx.doi org/10.1136/bmjopen-2018023337).

Received 5 April 2018

Revised 19 November 2018 Accepted 23 January 2019

Check for updates

(C) Author(s) (or their employer(s)) 2019. Re-use permitted under CC BY-NC. No commercial re-use. See rights and permissions. Published by BMJ.

${ }^{1}$ Research Institute for Primary Care and Health Sciences, Keele University, Newcastle, UK

${ }^{2}$ The Duke Clinical Research Institute, Durham, North Carolina, USA

${ }^{3}$ Department of Cardiology, University Hospital of Wales, Cardiff, UK

${ }^{4}$ Department of Health Sciences, University of Leicester, Leicester, UK

Correspondence to

Nafiu Ismail;

n.ismail@keele.ac.uk

\section{ABSTRACT}

Objective The primary objective was to determine the incidence of bleeding events post acute coronary syndrome (ACS) following hospital discharge. The secondary objective was to determine the prognostic impact of bleeding on mortality, major adverse cardiovascular events (MACE), myocardial re-infarction and rehospitalisation in the postdischarge setting.

Design A narrative systematic review.

Data source Medline, Embase, Amed and Central (Cochrane) were searched up to August 2018.

Study selection For the primary objective, randomised controlled trials (RCT) and observational studies reporting on the incidence of bleeding post hospital discharge were included. For the secondary objective, RCTs and observational studies that compared patients with bleeding versus those without bleeding post hospital discharge vis-à-vis mortality, MACE, myocardial re-infarction and rehospitalisation were included.

Results 53 studies ( 36 observational studies and 17 RCTs) with a combined cohort of 714458 participants for the primary objectives and 187317 for the secondary objectives were included. Follow-up ranged from 1 month to just over 4 years. The incidence of bleeding within 12 months post hospital discharge ranged from $0.20 \%$ to $37.5 \%$ in observational studies and between $0.96 \%$ and $39.4 \%$ in RCTs. The majority of bleeds occurred in the initial 3 months after hospital discharge with bruising the most commonly reported event. Major bleeding increased the risk of mortality by nearly threefold in two studies. One study showed an increased risk of MACE (HR $3.00,95 \% \mathrm{Cl}$ 2.75 to $3.27 ; p<0.0001$ ) with bleeding and another study showed a non-significant association with rehospitalisation (HR 1.20,95\% Cl 0.95 to 1.52; $p=0.13$ ).

Conclusion Bleeding complications following ACS management are common and continue to occur in the long term after hospital discharge. These bleeding complications may increase the risk of mortality and MACE, but greater evidence is needed to assess their longterm effects.

PROSPERO registration number CRD42017062378.

\section{INTRODUCTION}

The management of acute coronary syndrome (ACS) depends on the clinical
Strengths and limitations of this study

- This is the first systematic review that has examined the incidence and prognostic impacts of bleeding complications post acute coronary syndrome (ACS) within the outpatient setting.

- The review combined evidence from observational studies and randomised controlled trials involving a total of 714458 participants for the primary objectives and 187317 for the secondary objectives.

- The studies included in the review were heterogeneous in regard to bleeding definition, the ACS presentation, demographic characteristics of the study participants, severity and type of bleeding examined, length of follow-up, discharged antiplatelet and anticoagulant regimens, therefore we were unable to pool data quantitatively.

- The findings in relation to major adverse cardiovascular events and rehospitalisation should serve as hypothesis generating due to limited data.

presentation, with an overall aim of reducing myocardial ischaemia and adverse ischaemic events. ${ }^{1}$ This goal is fundamentally achieved via therapy with a combination of antithrombotic and invasive strategies. Paradoxically, these management strategies while achieving the desired goal of reducing ischaemic events increases the risk of bleeding complications. ${ }^{2-4}$ In the clinical trial setting, the incidence of major bleeding is reported to be between $1 \%$ and $10 \%$ depending on the bleeding definition used, ${ }^{5-7}$ with observational studies reporting incidences of between $2.8 \%^{8}$ and $11 \% .{ }^{9}$ However, the emphasis in the majority of these studies has been on major in-hospital or 30-day bleeding events (a composite of in-hospital and postdischarge events), with little consideration for events in the long term after hospital discharge. Post hospital discharge, patients with ACS may remain on dual antiplatelet therapy for up to a year, and 
aspirin indefinitely, so their risk of bleeding complications persist in the long term.

Major bleeding is an independent predictor of adverse outcomes, including mortality, recurrent myocardial infarction (MI), stroke, and stent thrombosis in patients with ACS. ${ }^{510-13}$ The association between major in-hospital bleeding events and adverse outcomes (most notably mortality) appeared to be maintained regardless of the definition of bleeding used. ${ }^{50-1214}$ These adverse events do, however, appear to depend on the anatomic site of the bleed,$^{15}$ and the site of bleeding may vary between the in-hospital and the postdischarge settings. While the nature of in-hospital bleeds and their association with adverse events has been well described, the timing, types and association of bleeding events that occur late after hospital discharge with clinical outcomes such as mortality is unclear.

To date, there has not been a systematic review of the incidence, types, and prognostic impact of bleeding events post hospital discharge for ACS. The primary objective of this systematic review was therefore to determine the incidence, timing, and types of post hospital discharge bleeds within the adult post-ACS population. The secondary objective was to determine the association of postdischarge bleeds with mortality, major adverse cardiovascular events (MACE), rehospitalisation and re-infarction in the outpatient setting.

\section{METHODS}

\section{Eligibility criteria}

There were two linked objectives for the systematic review. For the primary objective, we selected studies that reported on the incidence, timing, and types of bleeding post-ACS post hospital discharge. For the secondary objective, we included studies that compared patients with versus those without bleeding post-ACS post hospital discharge in relation to mortality, MACE, myocardial re-infarction and rehospitalisation. We only included randomised controlled trials (RCTs) where bleeding events were reported as secondary or safety outcomes, and observational studies which were published in English. Studies where the intervention was coronary artery bypass graft surgery or elective percutaneous coronary intervention (PCI) were excluded. We also excluded studies where the study population comprised patients with stable angina or other coronary artery disease. See table 1 for detailed inclusion and exclusion criteria for the review. For studies using the same data source, only one was included in the review, based on: (1) quality, and then by (2) sample size, followed by (3) length of follow-up, unless the studies reported on different outcomes.

\section{Search strategy}

Medline (Healthcare Databases Advanced Search (HDAS); 1946-August 2018), Embase (Ovid SP; 1974August 2018), Amed (Ovid SP; 1985-August 2018) and Central (Cochrane central register of controlled trials) were searched up to August 2018 using a search strategy which combined keywords and related database-specific subject headings for both primary and secondary objectives (see online supplementary table 1 for the full search strategy used on the Embase database). The Journal of the American College of Cardiology (JACC), the European Heart Journal, Heart, and Circulation were electronically searched for relevant articles and grey literature. The bibliographies of included studies and relevant review articles identified from each database were scrutinised for additional relevant articles. Citation tracking of included studies via Web of Science was carried out to retrieve additional relevant articles.

\section{Study selection}

The titles of all identified articles were screened and those which were obviously irrelevant were eliminated at this stage. The abstracts of the remaining articles were screened independently by NI and JP. Discordances were resolved by consensus between NI, JP and MAM. The full texts of the remaining articles were then screened by NI, with JP also screening 1 in 10.

\section{Data extraction}

We extracted study characteristics including study design, setting, length of follow-up, in-hospital interventions, participant characteristics, discharged therapy and comorbidities. The outcomes of incidence of postdischarge bleeding and associated 95\% CIs, time of bleed, location/type of bleed, and the adjusted and unadjusted associations of bleeding with mortality, MACE, re-infarction and rehospitalisation were extracted from individual studies onto a prepiloted and formatted spreadsheet. In studies where incidence and associated $95 \%$ CIs were not reported but relevant data were available, incidence per 100 persons at risk were calculated (ie, essentially as a proportion). For studies that combined in-hospital and postdischarge bleeds, and episodes of bleeds were stratified by time (for instance at 30 days, 6 months, 12 months), bleeds that occurred within the initial 30 days were considered to be in-hospital bleeds (decided by consensus of NI, KJP, MAM and UTK) and therefore removed from the numerator and denominator. The authors of original studies were contacted where necessary data were missing or to confirm methodological aspects or other characteristics of the study.

\section{Quality assessment}

Observational studies and post hoc observational analyses of RCTs were appraised by the Newcastle Ottawa Scale (NOS) for assessing risk of bias in non-randomised studies. ${ }^{16}$ The NOS quality assessment scale contains eight items partitioned into three categories of selection, comparability and outcome. A maximum of one star is allocated to a high-quality study for each item under selection and outcome and a maximum of two stars under comparability, giving an overall maximum of nine stars. We considered studies with an overall number 
Table 1 Inclusion and exclusion criteria specific to primary and secondary objectives

Inclusion criteria Exclusion criteria

Primary objective

- Participants aged 18years and over

$\checkmark$ Cannot be ascertained whether bleed occurred inhospital or postdischarge

- Participants discharged with an ACS diagnosis (UA or STEMI $\sqrt{ }$ In-hospital bleeds only or NSTEMI) at index hospitalisation

- Randomised controlled trial or observational study

$\checkmark$ Incidence and $95 \% \mathrm{Cl}$ or number of bleeding events cannot be extracted or calculated

Bleeding occurred after hospital discharge

$\checkmark$ Study population combined patients with ACS and other coronary diseases such as stable angina

- Any type of bleeding examined (such as gastrointestinal bleed) post hospital discharge for ACS

$\checkmark$ Postdischarge bleeding after $\mathrm{PCl}$, without specifying the clinical presentation for the $\mathrm{PCl}$ or whether the $\mathrm{PCl}$ was elective

\section{- Incidence and associated $95 \% \mathrm{Cl}$ can be extracted or calculated}

$\checkmark$ Only reporting CABG-related bleeds

$\checkmark$ Conference/study abstracts, editorials and reviews

\section{Secondary objective}

- Participants aged 18years and over

$\checkmark$ Cannot be ascertained whether bleed occurred inhospital or postdischarge

- Participants discharged with an ACS diagnosis (UA or STEMI $\sqrt{ }$ In-hospital bleeds only or NSTEMI) at index hospitalisation

- Randomised controlled trial or observational study

$\checkmark$ Study population combined patients with ACS and other coronary diseases such as stable angina

Bleeding occurred after hospital discharge

$\checkmark$ Postdischarge bleeding after $\mathrm{PCl}$, without specifying the clinical presentation for the $\mathrm{PCl}$ or whether the $\mathrm{PCl}$ was elective

- Evaluated outcome of or composite of mortality, MI, rehospitalisation and MACE in bleed vs no bleed cohorts

$\checkmark$ Only reporting CABG-related bleeds

$\checkmark$ Conference/study abstracts, editorials and reviews

ACS, acute coronary syndrome; CABG, coronary artery bypass graft; MACE, major adverse cardiovascular event; MI, myocardial infarction; NSTEMI, non-ST-elevation myocardial infarction; PCI, percutaneous coronary intervention; STEMI, ST-elevation myocardial infarction; UA, unstable angina.

of stars greater than or equal to six stars as high-quality studies. ${ }^{17}$ RCTs were appraised by the Scottish Intercollegiate Guideline Network quality assessment tool. ${ }^{18}$ Each study was categorised as high quality, acceptable quality or low quality based on the standard criteria for this tool. Quality assessment was based on the primary objective of each study as incidence of bleeding was typically reported as safety or secondary outcome measure.

\section{Data synthesis}

A narrative synthesis approach was applied due to heterogeneity in relation to length of follow-up, ACS presentation, definition of bleeding used, type of bleeding examined, severity of bleeding examined, geographical location and discharge therapy across studies. For the primary objective, the narrative synthesis was carried out in stages. Initially, the incidence of bleeding overall was summarised separately for observational studies and RCTs. The incidence of bleeding was then stratified by ACS presentation (ST-elevation myocardial infarction
[STEMI], non-ST-elevation myocardial infarction/ unstable angina [NSTEMI/UA]) and discharge antithrombotic drug combinations and duration (single antiplatelet [SAPT], dual antiplatelet [DAPT] and receipt of oral anticoagulant) in studies that reported these. To assess the incidence of bleeding by time from hospital discharge, the incidence of bleeding was stratified by follow-up time within studies which looked at multiple time periods. Where studies allowed, the incidence of bleeding stratified by major, minor and nuisance bleeds (see online supplementary table 2 for definitions), and the incidence of different types of bleeding events were examined.

We assessed the strength of evidence (SOE) for each secondary outcome following the Agency for Healthcare Research and Quality guideline. ${ }^{19}$ For each secondary outcome, assessment was carried out by examining risk of bias, consistency, directness and precision across studies that reported on this outcome, and a grade allocated 

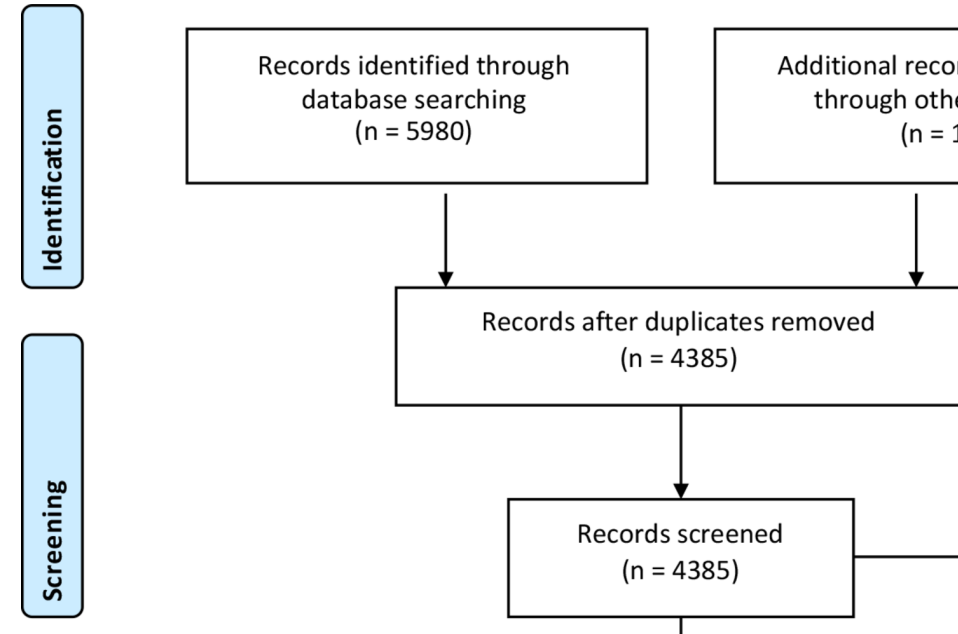

Additional records identified through other sources $(n=16)$
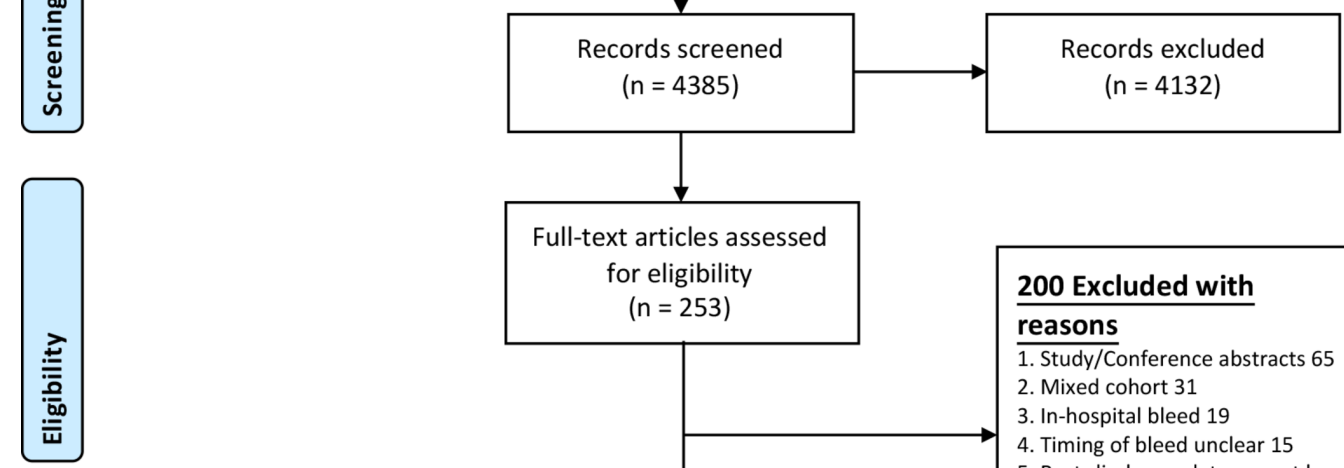

\section{Excluded with}

\section{reasons}

1. Study/Conference abstracts 65

2. Mixed cohort 31

3. In-hospital bleed 19

4. Timing of bleed unclear 15

5. Post-discharge data cannot be

extracted 31

6. Unclear cohort 3

7. Duplicate datasets 10

8. No accessible full text 10

9. Not written in English 5

Studies included in

qualitative synthesis

10. Reviews 5

11. Duplicate studies 4

$(n=53)$

12. webpage 1

13. Editorial 1

Figure 1 Preferred Reporting Items for Systematic Reviews and Meta-Analyses flow chart depicting steps involved in selecting or rejecting studies for inclusion in the review.

as high, moderate, low or insufficient based on these assessments.

\section{Patient and public involvement}

Patients and members of the public did not have any role in the design, conduct, data synthesis or reporting of the study.

\section{RESULTS}

The search of Medline, Embase, Amed and Central (Cochrane) identified 37 studies, ${ }^{20-56} 4$ studies were further identified from electronic search of JACC database, ${ }^{357-59} 2$ from Web of Science citation index, ${ }^{60} 619$ from bibliographic screening of included studies, ${ }^{4}$ 62-69 and finally, 1 from recommendation by an expert within the field. ${ }^{70}$ Overall, 53 studies (36 observational studies and 17 RCTs) were included in the review with a combined cohort of 714458 participants for the primary objectives and 187317 for the secondary objectives (figure 1). Of the 53 studies, 45 only reported on the primary outcomes, 3 only reported on the secondary outcomes and 5 reported on both primary and secondary outcomes.

\section{Characteristics of included studies}

The characteristics of included studies (for the primary objective) are summarised in table 2 for observational studies and table 3 for RCTs. Overall, 50 studies reported on the primary outcome, of which $68 \% \quad(n=34)$ were cohort studies and $32 \%(\mathrm{n}=16)$ were RCTs. The characteristics of included studies for the secondary objective are summarised in table 4. Overall, eight studies reported on the secondary outcomes, of which seven were cohort studies and one was an RCT.

Length of follow-up varied from 30 days $^{29}$ to just over 4 years ${ }^{69}$ post hospital discharge. The number of participants ranged from 193 to 187386 . The definition for bleeding used by each study in the review are provided in online supplementary table 2 . Some studies $(n=23)$ did not report bleeding events based on recognised definitions (such as Bleeding Academic Research Consortium [BARC]). Of the included studies, 27 had specified the in-hospital ACS management strategy. In 26 of these studies, PCI was the baseline management strategy, and in one study the management strategy was a combination of PCI, angiography and medical therapy. 


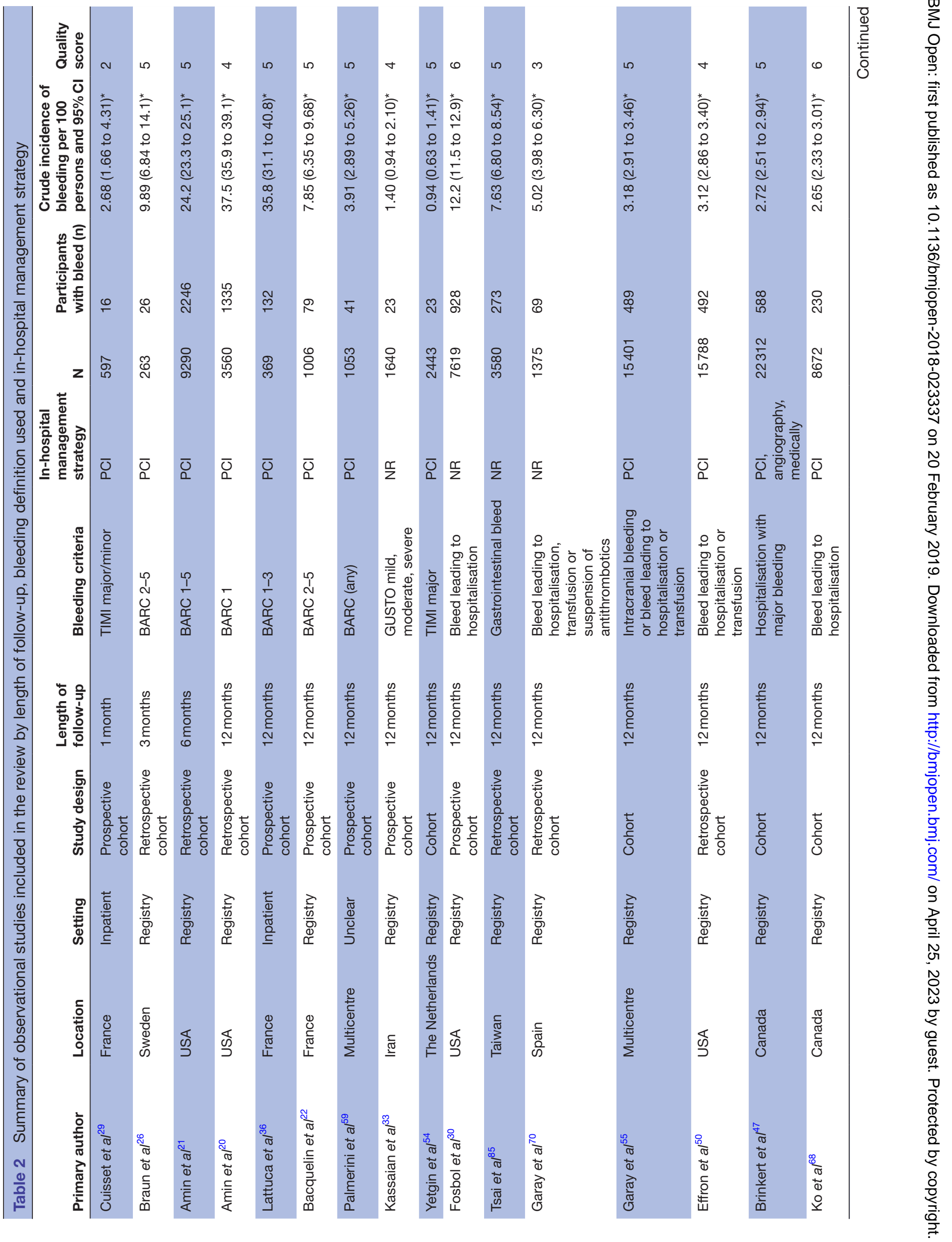




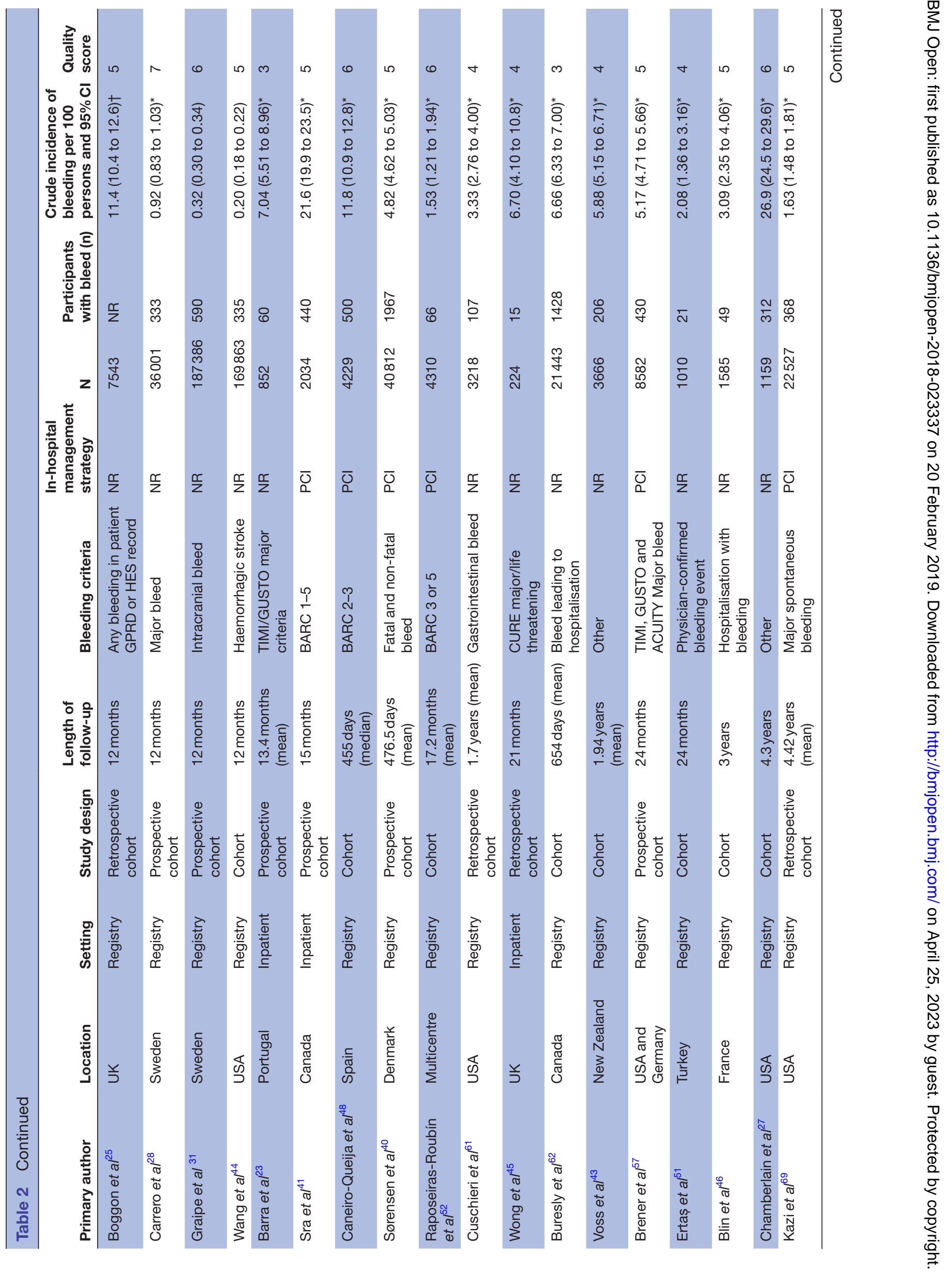




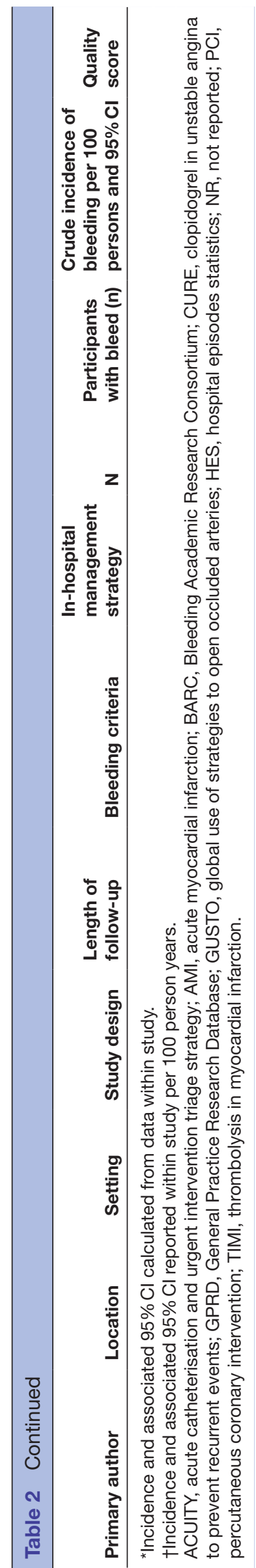

Risk of bias assessment

Summaries of risk of bias of individual studies are provided in tables 2,3 and 4. Sixty-nine per cent $(n=25)$ of the observational studies were at high risk of bias due to lack of reporting on presence/absence of outcome at start of study, attrition rate, and comparability of cohorts based on analysis (whether study adjusted for confounders or not). Thirty-one per cent $(n=11)$ were at low risk of bias. Two RCTs were high risk, four were at an acceptable risk of bias and two were low risk. The main reasons for low quality in RCTs were inadequate reporting on randomisation, concealment, blinding, adequacy and reliability of outcome measurements. For studies which were post hoc observational analysis of RCTs, five were high risk and four were at low risk of bias.

\section{Incidence of bleeding}

In a cohort of 611412 participants, 14217 (2.3\%) episodes of bleeds were reported in 34 observational studies and $2685(2.6 \%)$ episodes in a cohort of 103046 participants in 16 RCTs (714458 participants overall). A summary of the incidence from each study is presented by length of follow-up, bleeding definition used and in-hospital management strategy in table 2 for observational studies and table 3 for RCTs. The overall incidence of bleeding within 12 months post hospital discharge varied from $0.2 \%{ }^{44}$ to $37.5 \%^{20}$ in observational studies, and between $0.96 \%{ }^{42}$ and $39.4 \%{ }^{63}$ in RCTs.

The incidence of bleeding stratified by ACS presentation (STEMI, NSTEMI/UA) and discharge antithrombotic drug combinations and duration (SAPT, DAPT and receipt of oral anticoagulant) are summarised by length of follow-up and the bleeding definition used in online supplementary tables 3, 4 and 5. Among those discharged on DAPT with aspirin and a thienopyridine, the incidence of bleeding within the first 12 months based on BARC criteria ranged from $3.91 \%$ to $38.8 \%$ (see online supplementary table 4) in observational studies, and between $0.96 \%$ and $47.4 \%$ in RCTs (see online supplementary table 5 ).

Eight observational studies 2336454751545970 and two RCT $^{56} 58$ comprising 53318 participants reported bleeding episodes at different time points during follow-up. In these studies, around one-half of bleeds that occurred in the first year post hospital discharge for ACS happened in the initial 1-3 months (figure 2).

The incidence of major bleeding events in observational studies (based on BARC 3-5) within the first 12 months of hospital discharge was around $1.29 \%-3.25 \%$. The incidence of minor bleeding events (based on BARC 2) and nuisance bleeds (based on BARC 1) within the same period were around $6.56 \%-10.6 \%$ and $21.9 \%-37.5 \%$, respectively (see figure 3 and online supplementary table 6). Generally, bruising (defined as skin haematoma, ecchymosis, petechiae) were the most commonly reported types of bleeding events post hospital discharge (range: $1.49 \%-22.5 \%$ within 12 months) followed by 


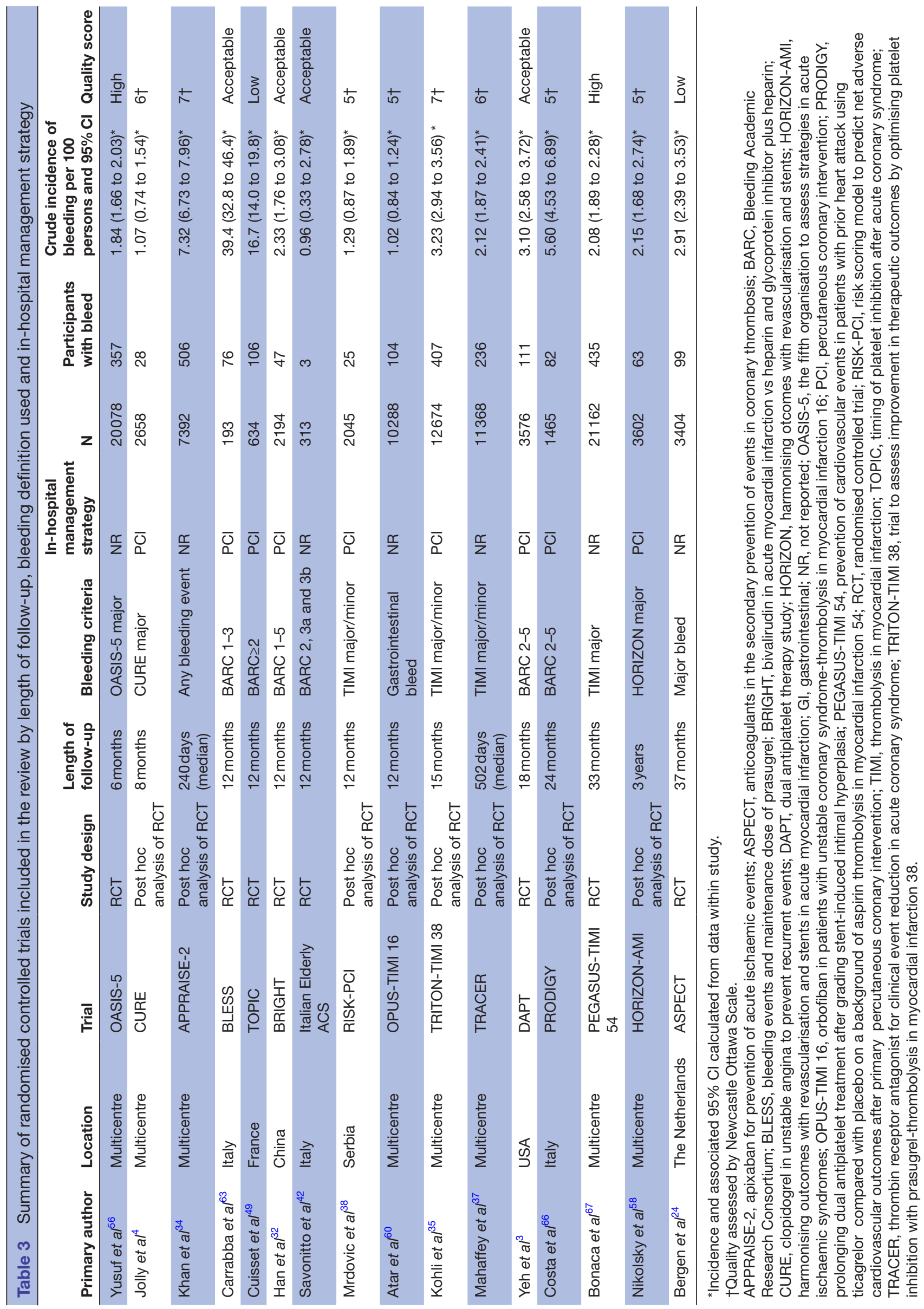




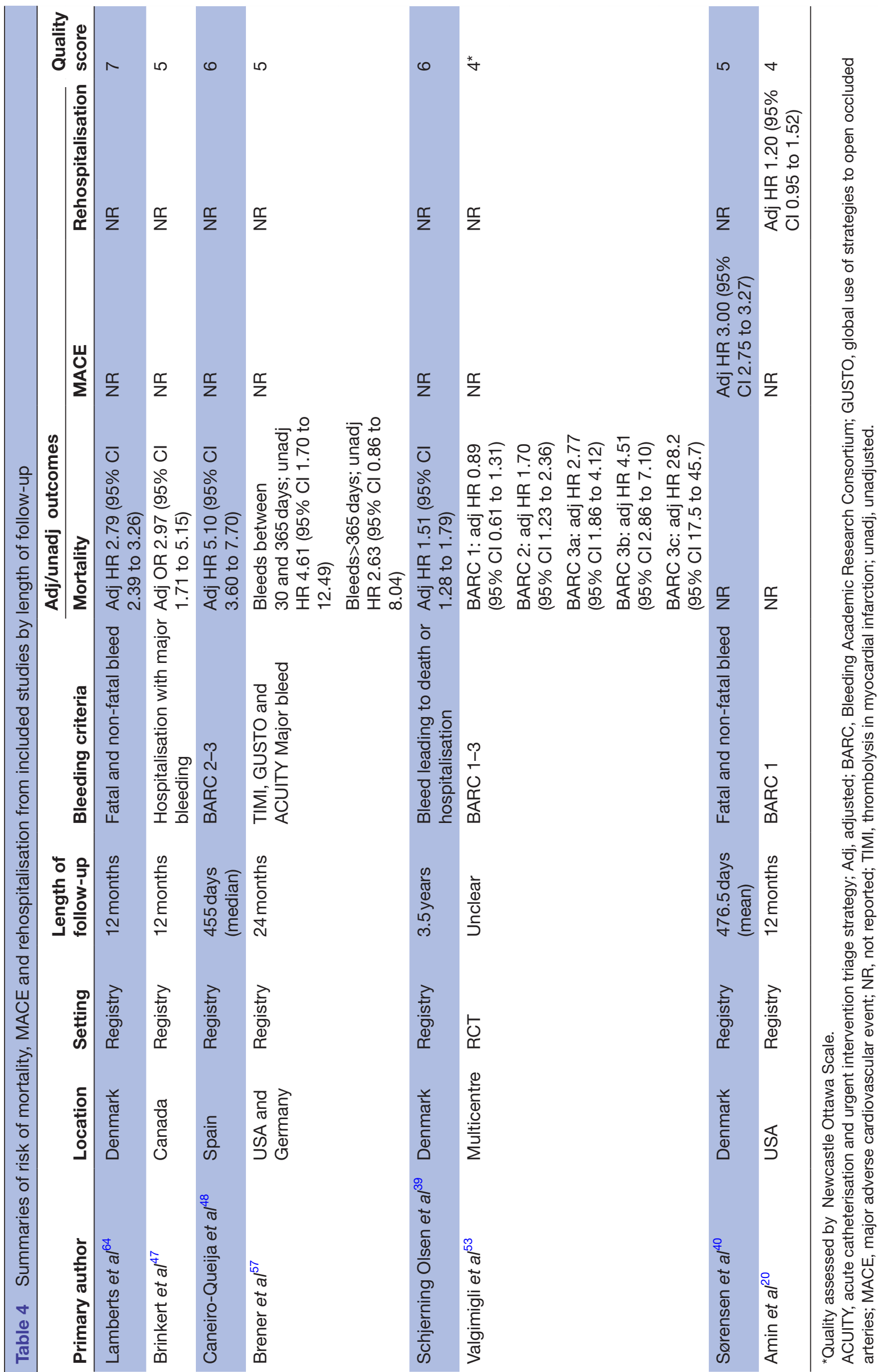




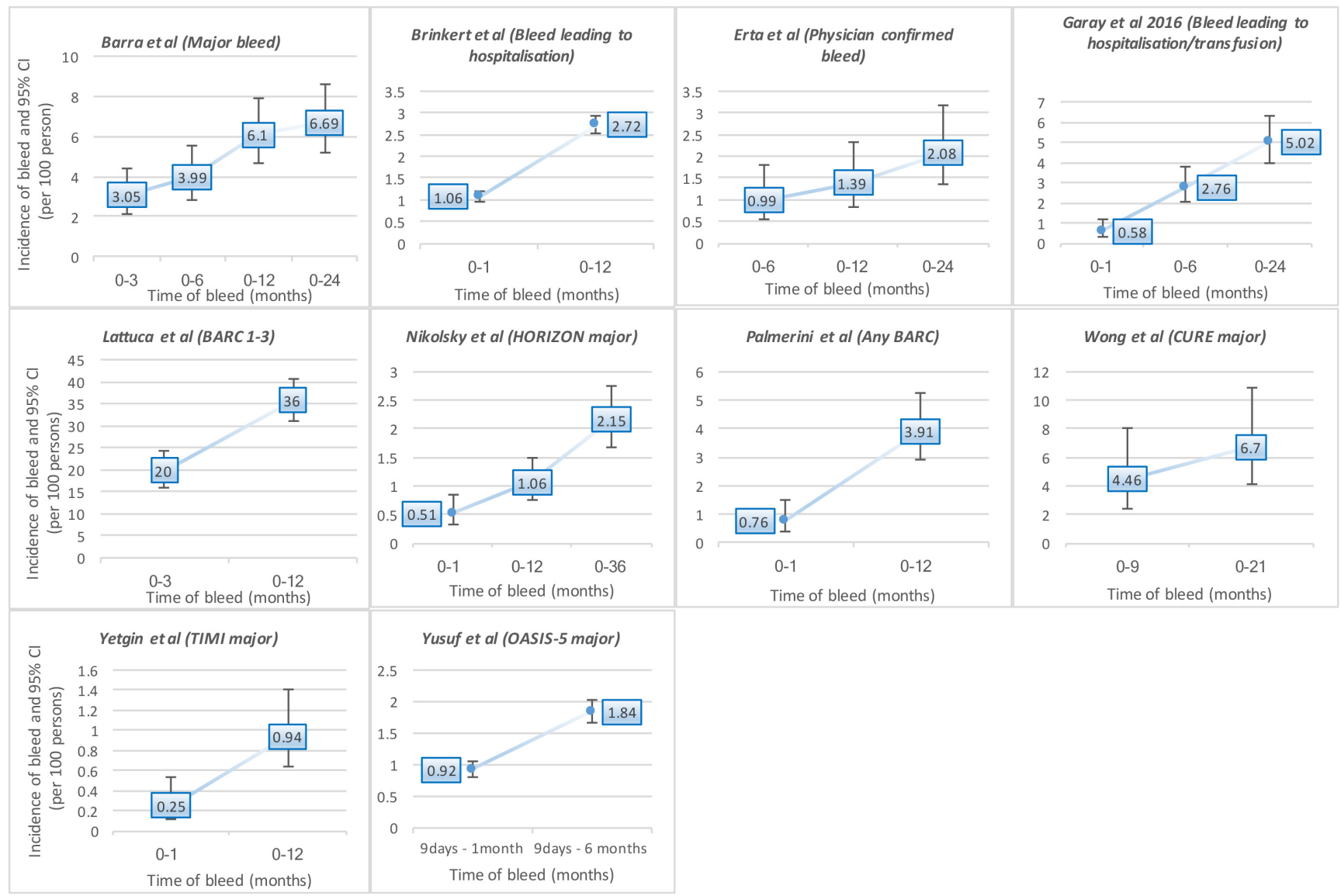

Figure 2 Cumulative incidence of bleeding as reported within individual studies at different time points (incidence expressed as proportion per 100 persons). BARC, Bleeding Academic Research Consortium; CURE, clopidogrel in unstable angina to prevent recurrent events; HORIZON, harmonising outcomes with revascularisation and stents; OASIS-5, the fifth organisation to assess strategies in acute ischaemic syndromes; TIMI, thrombolysis in myocardial infarction.

gastrointestinal bleeds (range: $0.25 \%-7.63 \%$ within 12 months; see figure 4 and online supplementary table 7 ).

\section{Bleeding and risk of mortality}

There was consistent reporting of an association between postdischarge bleeding and all-cause mortality in five observational studies ${ }^{3947485764}$ and one RCT ${ }^{53}$ (table 4). Major bleeding was associated with nearly threefold increased risk of mortality in the first 12 months of hospital discharge in two studies (table 4) ${ }^{4764}$ Nuisance bleeding events defined as BARC 1 were not associated with mortality in one RCT, but there was an increased risk of mortality with BARC 2 and 3 bleeds in the same RCT, ${ }^{53}$ which increased with bleeding severity (table 4). The SOE for the outcome of mortality was rated low (online supplementary table 8 ).

\section{Bleeding and risk of MACE, rehospitalisation and re-infarction} The adjusted (adj) risk (HR) of MACE with bleeding (defined as bleeds leading to hospitalisation or death) was 3.00 (95\% CI 2.75 to 3.27 in one study; table 4 ). ${ }^{40}$ There was a statistically non-significant association between postdischarge bleed (defined as BARC 1 bleeds) and risk of rehospitalisation (adj HR 1.20, 95\% CI 0.95 to 1.52 in another study; table 4$).{ }^{20}$ There were no studies examining the association between postdischarge bleeding and subsequent risk of re-infarction. The SOE for the outcomes of MACE and rehospitalisation were rated insufficient (online supplementary table 8 ).

\section{DISCUSSION}

Our systematic review is the first to study the incidence, timing and types of postdischarge bleeding complications, and their association with mortality, MACE, re-infarction and rehospitalisation. Fifty-three studies were included, comprising 36 observational studies and 17 RCTs with a combined cohort of 714458 participants for the primary objectives and 187317 for the secondary objectives. We report that bleeding complications post-ACS are common following hospital discharge, and vary by length of follow-up, severity, type and the definition of bleeding used. We report that the incidence of bleeding was highest in the initial 3 months after hospital discharge for ACS, with bleeding events continuing to occur even after 1-year postdischarge. The majority of postdischarge bleeding events were nuisance bleeds such as ecchymosis and petechiae, with major bleeding events such as intracranial haemorrhage less common. While there was 


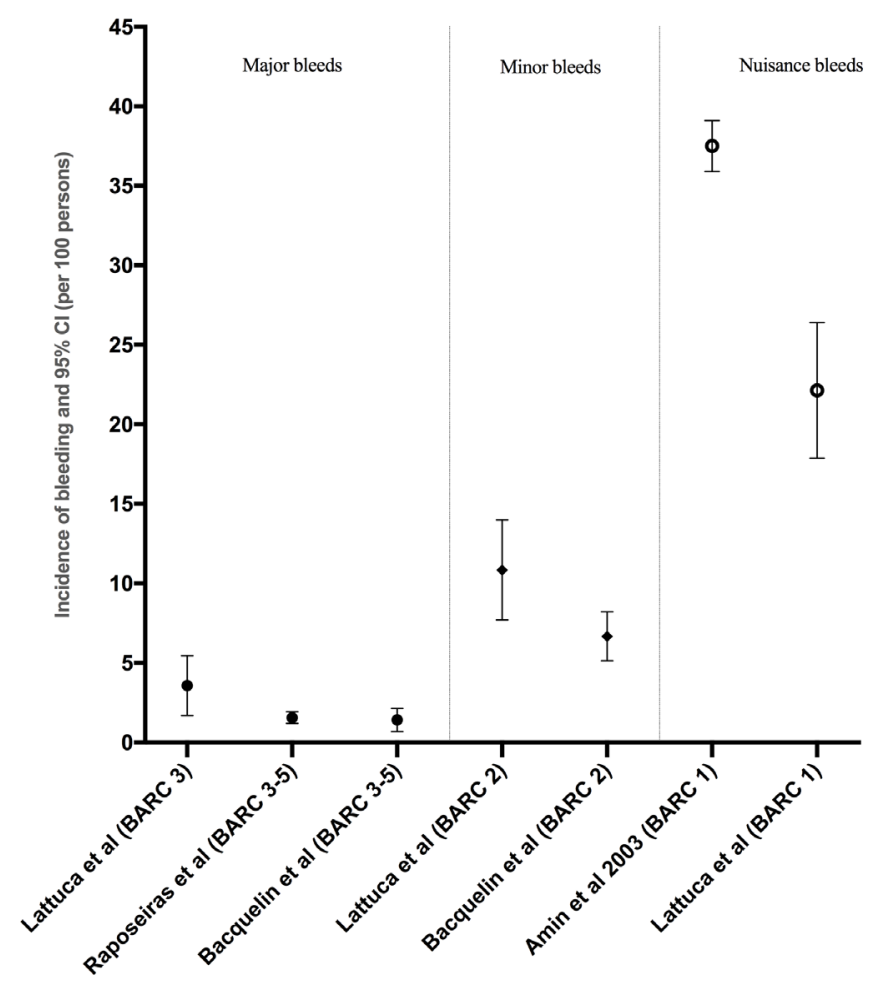

Figure 3 Incidence of bleeding stratified by severity in observational studies that reported bleeding by Bleeding Academic Research Consortium (BARC) criteria within the first 12 months after hospital discharge.

substantial heterogeneity between studies, we report that up to one-third of patients discharged on DAPT will experience bleeding complications in the first 12 months after hospital discharge, and around $1.3 \%-3.3 \%$ of patients will experience a major bleed.

Our review shows that major bleeding may increase the risk of mortality by nearly threefold in the first 12 months after hospital discharge, but the strength of the evidence was weak. We identified very limited data on whether postdischarge bleeding was associated with MACE and rehospitalisation. Although there was an indication of

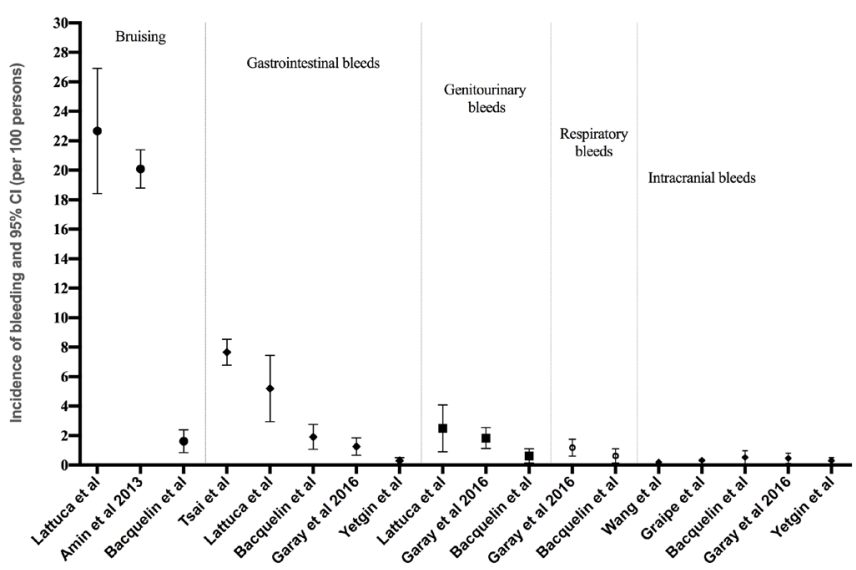

Figure 4 Incidence of each type of bleeding event within the first 12 months after hospital discharge in observational studies. an association with MACE in one study ${ }^{40}$ and rehospitalisation in another, ${ }^{20}$ the latter association did not reach statistical significance.

\section{Clinical implications}

Although current guidelines ${ }^{71-73}$ have recommended dual therapy with aspirin and a thienopyridine for up to 12 months and triple therapy in the presence of comorbid conditions such as atrial fibrillation for shorter periods, it was evident from our study that these maintenance therapies are accompanied by bleeding complications which predominantly occur post hospital discharge. Consideration must therefore be given to ways of minimising these bleeding complications, such as by encouraging clinicians to use risk scoring algorithms such as DAPT, ${ }^{74}$ precise-DAPT, ${ }^{75}$ BleeMACS score ${ }^{76}$ (for patients with ACS treated with PCI) or TRILOGY-ACS bleeding risk model ${ }^{77}$ (for patients with NSTEMI/UA managed medically) to identify patients at higher risk of these bleeding complications, such that maintenance oral antithrombotics or newer oral anticoagulants that have more favourable safety profile than warfarin can be tailored to fit each patient's risk profile. However, it must be borne in mind that many of these risk algorithms were developed in the clinical trial setting, and have not yet been validated in unselected cohorts. Aspirin regardless of dose increases the risk of gastrointestinal bleeds. ${ }^{78}{ }^{79}$ In high-risk patients such as those with previous history of these types of bleeds, concomitant use of a proton pump inhibitor as advocated by the European Society of Cardiology guidelines will reduce the future risk of these bleeds. ${ }^{80}$

\section{Research implications}

The majority of studies in this review were not primarily designed to investigate the incidence of bleeding complications. This meant that incidences could only be reported here as per 100 persons, that is, essentially as a proportion, rather than per 100 persons years at risk. This underscores the need to examine the incidence of these bleeding events using high-quality observational studies that are more reflective of the real-world populations encountered in clinical practice. The incidence of postdischarge bleeding complications may vary by type of bleed, patient demographics and discharge pharmacotherapy. Future studies should explore factors associated with postdischarge bleeding complications so that risk stratification tools that are more representative of the unselected cohorts encountered in clinical practice (often ignored in RCTs) can be developed to identify individuals at high risk of bleeding post hospital discharge, as most contemporary bleeding risk scores predict in-hospital bleeding events. ${ }^{81-83}$

We also report that bleeding complications post hospital discharge may be associated with subsequent risk of mortality, although evidence from the literature was limited. The risks of MACE and rehospitalisation were only reported in two studies, and none of the studies in the review reported on re-infarction. Future research is 
required to quantify these associations, with particular emphasis on whether nuisance and minor bleeding events that are much more common post hospital discharge also have a prognostic impact. Finally, future research examining these associations should stratify by the timing of the bleeding events in order to determine whether the prognostic impacts of these bleeding complications are more pronounced in the early phases of hospital discharge or are equally important in the long term after hospital discharge.

\section{Limitations of the review}

This study has several potential limitations. First, the studies included in the review were too heterogeneous in regard to bleeding definition, ACS presentation, demographic characteristics of the study participants, severity and type of bleeding examined, length of follow-up, discharge antiplatelet and anticoagulant regimens to pool data to obtain an overall incidence and mortality figures. Second, the duration and dosage of discharge antithrombotic therapy as well as in-hospital management strategies were not specified in the majority of studies (due to selective reporting), as such we were unable to adequately assess the impact of these factors on the incidence of bleeding. In the majority of studies, episodes of bleeds were extracted to calculate incidence figures. In most of these studies, there was lack of clarity on whether patients were included in the numerator more than once if they had multiple episodes of bleeds. However, since bleeding complications are rare events ${ }^{584}$ and having more than one episode of bleeding is even rarer, it is unlikely that this would have affected the overall incidence. Similarly, for some studies where episodes of bleeds were reported at different time intervals and the number of people at risk within each time interval were not reported, incidence figures were estimated based on the assumption that there was no attrition, hence these figures may have been underestimated.

\section{CONCLUSIONS}

In this systematic review of 53 studies, bleeding complications post hospital discharge for ACS were found to be common, with bruising the most common. These bleeding complications vary by severity, anatomic source and type of discharge antithrombotic therapy, and while most common immediately postdischarge, these bleeds continue to occur in the long term. There are limited data around the long-term outcomes of patients that sustain bleeding events post hospital discharge for ACS. Further work is required to define the nature, frequency and prognostic impact of such bleeding events, using formal bleeding definitions. Real-world risk stratification tools will need to be developed that specifically predict the risk of bleeding complications postdischarge to identify highrisk individuals for a more patient-centred approach in managing optimal pharmacotherapy and care.
Acknowledgements The authors would like to thank Joanne L Jordan, Nadia Corp and Opeyemi Babatunde of the Research Institute for Primary Care and Health Sciences for their support in drafting and conducting the review.

Contributors NI designed the study under the supervision of KJP, MAM, UTK. NI and JP conducted the study and analysed the data. NI wrote the first draft and KJP, MAM, UTK, TK and SR made critical revision of the manuscript. All authors have approved the final manuscript and KJP, MAM and UTK are the guarantors.

Funding This study was funded by North Staffordshire Medical Institute 50th Anniversary Award.

Disclaimer The funding body did not have any role in the design, conduct, data synthesis or reporting of the study.

Competing interests None declared.

Patient consent for publication Not required.

Provenance and peer review Not commissioned; externally peer reviewed.

Data sharing statement The dataset, supplementary appendix and review protocol are available from the corresponding author at n.ismail@keele.ac.uk.

Open access This is an open access article distributed in accordance with the Creative Commons Attribution Non Commercial (CC BY-NC 4.0) license, which permits others to distribute, remix, adapt, build upon this work non-commercially, and license their derivative works on different terms, provided the original work is properly cited, appropriate credit is given, any changes made indicated, and the use is non-commercial. See: http://creativecommons.org/licenses/by-nc/4.0/.

\section{REFERENCES}

1. O'Connor RE, Brady W, Brooks SC, et al. Part 10: Acute Coronary Syndromes. Circulation 2010;122.

2. Cayla G, Cuisset T, Silvain J, et al. Prasugrel monitoring and bleeding in real world patients. Am J Cardiol 2013;111:38-44.

3. Yeh RW, Kereiakes DJ, Steg PG, et al. Benefits and Risks of Extended Duration Dual Antiplatelet Therapy After PCl in Patients With and Without Acute Myocardial Infarction. J Am Coll Cardiol 2015;65:2211-21.

4. Jolly SS, Pogue J, Haladyn K, et al. Effects of aspirin dose on ischaemic events and bleeding after percutaneous coronary intervention: insights from the PCI-CURE study. Eur Heart $J$ 2009;30:900-7.

5. Rao SV, Eikelboom JA, Granger CB, et al. Bleeding and blood transfusion issues in patients with non-ST-segment elevation acute coronary syndromes. Eur Heart J 2007;28:1193-204.

6. Mehta SR, Granger CB, Eikelboom JW, et al. Efficacy and safety of fondaparinux versus enoxaparin in patients with acute coronary syndromes undergoing percutaneous coronary intervention: results from the OASIS-5 trial. J Am Coll Cardiol 2007;50:1742-51.

7. Doyle BJ, Rihal CS, Gastineau DA, et al. Bleeding, blood transfusion, and increased mortality after percutaneous coronary intervention: implications for contemporary practice. J Am Coll Cardiol 2009;53:2019-27.

8. Spencer FA, Moscucci M, Granger CB, et al. Does comorbidity account for the excess mortality in patients with major bleeding in acute myocardial infarction? Circulation 2007;116:2793-801.

9. Amlani S, Nadarajah T, Afzal R, et al. Mortality and morbidity following a major bleed in a registry population with acute ST elevation myocardial infarction. J Thromb Thrombolysis 2010;30:434-40.

10. Eikelboom JW, Mehta SR, Anand SS, et al. Adverse Impact of Bleeding on Prognosis in Patients With Acute Coronary Syndromes. Circulation 2006;114:114-782.

11. Manoukian SV, Feit F, Mehran R, et al. Impact of major bleeding on 30-day mortality and clinical outcomes in patients with acute coronary syndromes: an analysis from the ACUITY Trial. J Am Coll Cardiol 2007;49:1362-8.

12. Rao SV, O'Grady K, Pieper KS, et al. Impact of bleeding severity on clinical outcomes among patients with acute coronary syndromes. Am J Cardiol 2005;96:1200-6.

13. Kinnaird TD, Stabile E, Mintz GS, et al. Incidence, predictors, and prognostic implications of bleeding and blood transfusion following percutaneous coronary interventions. Am J Cardiol 2003;92:930-5.

14. Kwok CS, Rao SV, Myint PK, et al. Major bleeding after percutaneous coronary intervention and risk of subsequent mortality: a systematic review and meta-analysis. Open Heart 2014;1:e000021.

15. Kwok CS, Khan MA, Rao SV, et al. Access and non-access site bleeding after percutaneous coronary intervention and risk of 
subsequent mortality and major adverse cardiovascular events: systematic review and meta-analysis. Circ Cardiovasc Interv 2015;8.

16. Wells GA, Shea B, O'Connell D, et al. The Newcastle-Ottawa Scale (NOS) for assessing the quality of nonrandomised studies in metaanalyses. 2011

17. Zhu W, Wan R, Liu F, et al. Relation of Body Mass Index With Adverse Outcomes Among Patients With Atrial Fibrillation: A MetaAnalysis and Systematic Review. J Am Heart Assoc 2016;5.

18. SIGN. Scottish Intercollegiate Guideline Network). Critical appraisal: Notes and checklists.

19. Owens DK, Lohr KN, Atkins D, et al. AHRQ series paper 5: grading the strength of a body of evidence when comparing medical interventions--agency for healthcare research and quality and the effective health-care program. J Clin Epidemiol 2010;63:513-23.

20. Amin AP, Bachuwar A, Reid KJ, et al. Nuisance bleeding with prolonged dual antiplatelet therapy after acute myocardial infarction and its impact on health status. J Am Coll Cardiol 2013;61:2130-8.

21. Amin AP, Wang TY, McCoy L, et al. Impact of Bleeding on Quality of Life in Patients on DAPT: Insights From TRANSLATE-ACS. J Am Coll Cardiol 2016;67:59-65.

22. Bacquelin R, Oger E, Filippi E, et al. Safety of prasugrel in real-world patients with ST-segment elevation myocardial infarction: 1-year results from a prospective observational study (Bleeding and Myocardial Infarction Study). Arch Cardiovasc Dis 2016;109:31-8.

23. Barra S, Providência R, Caetano F, et al. BLEED-Myocardial Infarction Score: Predicting mid-term post-discharge bleeding events. World J Cardiol 2013:5:196-206.

24. Effect of long-term oral anticoagulant treatment on mortality and cardiovascular morbidity after myocardial infarction. Anticoagulants in the Secondary Prevention of Events in Coronary Thrombosis (ASPECT) Research Group. Lancet 1994;343:499-503

25. Boggon R, van Staa TP, Timmis A, et al. Clopidogrel discontinuation after acute coronary syndromes: frequency, predictors and associations with death and myocardial infarction--a hospital registry-primary care linked cohort (MINAP-GPRD). Eur Heart $J$ 2011;32:2376-86.

26. Braun OÖ, Bico B, Chaudhry U, et al. Concomitant use of warfarin and ticagrelor as an alternative to triple antithrombotic therapy after an acute coronary syndrome. Thromb Res 2015;135:26-30.

27. Chamberlain AM, Gersh BJ, Mills RM, et al. Antithrombotic strategies and outcomes in acute coronary syndrome with atrial fibrillation. Am J Cardiol 2015;115:1042-8.

28. Carrero JJ, Varenhorst C, Jensevik K, et al. Long-term versus shortterm dual antiplatelet therapy was similarly associated with a lower risk of death, stroke, or infarction in patients with acute coronary syndrome regardless of underlying kidney disease. Kidney Int 2017;91:216-26.

29. Cuisset T, Cayla G, Frere C, et al. Predictive value of post-treatment platelet reactivity for occurrence of post-discharge bleeding after non-ST elevation acute coronary syndrome. Shifting from antiplatelet resistance to bleeding risk assessment? Eurolntervention 2009;5:325-9.

30. Fosbol EL, Wang TY, Li S, et al. Safety and effectiveness of antithrombotic strategies in older adult patients with atrial fibrillation and non-ST elevation myocardial infarction. Am Heart $J$ 2012;163:720-8.

31. Graipe A, Binsell-Gerdin E. S€ Oderstr€ Om L, Mooe T. Incidence, Time Trends, and Predictors of Intracranial Hemorrhage During LongTerm Follow-up After Acute Myocardial Infarction.

32. Han Y, Guo J, Zheng Y, et al. Bivalirudin vs heparin with or without tirofiban during primary percutaneous coronary intervention in acute myocardial infarction: the BRIGHT randomized clinical trial. JAMA 2015;313:1336.

33. Kassaian SE, Masoudkabir F, Sezavar H, et al. Clinical characteristics, management and 1-year outcomes of patients with acute coronary syndrome in Iran: the Iranian Project for Assessment of Coronary Events 2 (IPACE2). BMJ Open 2015;5:e007786.

34. Khan R, Lopes RD, Neely ML, et al. Characterising and predicting bleeding in high-risk patients with an acute coronary syndrome. Heart 2015;101:1475-84.

35. Kohli P, Udell JA, Murphy SA, et al. Discharge Aspirin Dose and Clinical Outcomes in Patients With Acute Coronary Syndromes Treated With Prasugrel Versus Clopidogrel. J Am Coll Cardiol 2014;63:225-32.

36. Lattuca B, Fabbro-Peray P, Leclercq F, et al. One-year incidence and clinical impact of bleeding events in patients treated with prasugrel or clopidogrel after ST-segment elevation myocardial infarction. Arch Cardiovasc Dis 2016;109:337-47.

37. Mahaffey KW, Huang Z, Wallentin L, et al. Association of aspirin dose and vorapaxar safety and efficacy in patients with non-ST-segment elevation acute coronary syndrome (from the TRACER Trial). Am J Cardiol 2014;113:936-44.

38. Mrdovic I, Savic L, Asanin M, et al. Sex-related analysis of short- and long-term clinical outcomes and bleeding among patients treated with primary percutaneous coronary intervention: an evaluation of the RISK-PCI data. Can J Cardiol 2013;29:1097-103.

39. Schjerning Olsen AM, Gislason GH, McGettigan P, et al. Association of NSAID use with risk of bleeding and cardiovascular events in patients receiving antithrombotic therapy after myocardial infarction. JAMA 2015;313:805.

40. Sørensen R, Hansen ML, Abildstrom SZ, et al. Risk of bleeding in patients with acute myocardial infarction treated with different combinations of aspirin, clopidogrel, and vitamin $\mathrm{K}$ antagonists in Denmark: a retrospective analysis of nationwide registry data. Lancet 2009;374:1967-74

41. Sra S, Tan MK, Mehta SR, et al. Ischemic and bleeding events in patients with myocardial infarction undergoing percutaneous coronary intervention who require oral anticoagulation: Insights from the Canadian observational AntiPlatelet sTudy. Am Heart $J$ 2016;180:82-9.

42. Savonitto S, Cavallini C, Petronio AS, et al. Early aggressive versus initially conservative treatment in elderly patients with nonST-segment elevation acute coronary syndrome: a randomized controlled trial. JACC Cardiovasc Interv 2012;5:906-16.

43. Voss WB, Lee M, Devlin GP, et al. Incidence and type of bleeding complications early and late after acute coronary syndrome admission in a New Zealand cohort (ANZACS-QI-7). N Z Med J 2016;129:27-38

44. Wang Y, Lichtman JH, Dharmarajan K, et al. National trends in stroke after acute myocardial infarction among Medicare patients in the United States: 1999 to 2010. Am Heart J 2015;169:78-85.

45. Wong P, Robinson A, Shaw S, et al. Long term clinical outcome and bleeding complications among hospital survivors with acute coronary syndromes. Postgrad Med J 2006;82:224-7.

46. Blin P, Dureau-Pournin C, Lassalle R, et al. Outcomes in patients after myocardial infarction similar to those of the PEGASUS-TIMI 54 trial: A cohort study in the French national claims database. $\mathrm{Br} J$ Clin Pharmacol 2017;83:2056-65.

47. Brinkert M, Southern DA, James MT, et al. Incidence and Prognostic Implications of Late Bleeding After Myocardial Infarction or Unstable Angina According to Treatment Strategy. Can J Cardiol 2017;33:998-1005

48. Caneiro-Queija B, Abu-Assi E, Raposeiras-Roubín S, et al. Differential Prognostic Impact on Mortality of Myocardial Infarction Compared With Bleeding Severity in Contemporary Acute Coronary Syndrome Patients. Rev Esp Cardiol 2018;71:829-36.

49. Cuisset T, Deharo P, Quilici J, et al. Benefit of switching dual antiplatelet therapy after acute coronary syndrome: the TOPIC (timing of platelet inhibition after acute coronary syndrome) randomized study. Eur Heart J 2017;38:3070-8.

50. Effron MB, Nair KV, Molife C, et al. One-Year Clinical Effectiveness Comparison of Prasugrel with Ticagrelor: Results from a Retrospective Observational Study using an Integrated Claims Database. Am J Cardiovasc Drugs 2018:18:129-41.

51. Ertaș FS, Tokgozoglu L. EPICOR Study Group. Long-term follow-up of antithrombotic management patterns in acute coronary syndrome patients. Turk Kardiyol Dern Ars 2018;46:175-83.

52. Raposeiras-Roubín S, Caneiro Queija B, D'Ascenzo F, et al. Usefulness of the PARIS Score to Evaluate the Ischemic-hemorrhagic Net Benefit With Ticagrelor and Prasugrel After an Acute Coronary Syndrome. Rev Esp Cardiol 2018

53. Valgimigli M, Costa F, Lokhnygina $Y$, et al. Trade-off of myocardia infarction vs. bleeding types on mortality after acute coronary syndrome: lessons from the Thrombin Receptor Antagonist for Clinical Event Reduction in Acute Coronary Syndrome (TRACER) randomized trial. Eur Heart J 2017;38:ehw525.

54. Yetgin T, Boersma E, Smits PC, et al. One-year efficacy and safety of routine prasugrel in patients with acute coronary syndromes treated with percutaneous coronary intervention: results of the prospective rijnmond collective cardiology research study. Neth Heart $J$ 2018;26(7-8):393-400.

55. Garay A, Ariza-Solé A, Formiga F, et al. Prediction of post-discharge bleeding in elderly patients with acute coronary syndromes: insights from the BleeMACS registry. Thromb Haemost 2018;118:929-38.

56. Yusuf S, Mehta SR, Chrolavicius S, et al. Comparison of fondaparinux and enoxaparin in acute coronary syndromes. $N$ Engl J Med 2006;354:1464-76.

57. Brener SJ, Kirtane AJ, Stuckey TD, et al. The Impact of Timing of Ischemic and Hemorrhagic Events on Mortality After Percutaneous Coronary Intervention: The ADAPT-DES Study. JACC Cardiovasc Interv 2016:9:1450-7. 
58. Nikolsky E, Mehran R, Dangas GD, et al. Cerebrovascular events after a primary percutaneous coronary intervention strategy for acute ST-segment-elevation myocardial infarction: analysis from the HORIZONS-AMI Trial. Circ Cardiovasc Interv 2015;8:e002283.

59. Palmerini $T$, Calabrò $P$, Piscione $F$, et al. Impact of gene polymorphisms, platelet reactivity, and the SYNTAX score on 1 -year clinical outcomes in patients with non-ST-segment elevation acute coronary syndrome undergoing percutaneous coronary intervention: the GEPRESS study. JACC Cardiovasc Interv 2014;7:1117-27.

60. Atar S, Cannon CP, Murphy SA, et al. Statins are associated with lower risk of gastrointestinal bleeding in patients with unstable coronary syndromes: Analysis of the Orbofiban in Patients with Unstable coronary Syndromes-Thrombolysis In Myocardial Infarction 16 (OPUS-TIMI 16) trial. Am Heart J 2006;151:976.e1-6.

61. Cuschieri JR, Drawz P, Falck-Ytter Y, et al. Risk factors for acute gastrointestinal bleeding following myocardial infarction in veteran patients who are prescribed clopidogrel. J Dig Dis 2014;15:195-201.

62. Buresly K, Eisenberg MJ, Zhang X, et al. Bleeding complications associated with combinations of aspirin, thienopyridine derivatives, and warfarin in elderly patients following acute myocardial infarction. Arch Intern Med 2005;165:784.

63. Carrabba N, Parodi G, Marcucci R, et al. Bleeding events and maintenance dose of prasugrel: BLESS pilot study. Open Heart 2016;3:e000460

64. Lamberts M, Gislason GH, Olesen JB, et al. Oral anticoagulation and antiplatelets in atrial fibrillation patients after myocardial infarction and coronary intervention. J Am Coll Cardiol 2013;62:981-9.

65. Tsai Y-W, Wen Y-W, Huang W-F, et al. Cardiovascular and gastrointestinal events of three antiplatelet therapies: clopidogrel, clopidogrel plus proton-pump inhibitors, and aspirin plus protonpump inhibitors in patients with previous gastrointestinal bleeding. $J$ Gastroenterol 2011;46:39-45.

66. Costa F, Vranckx P, Leonardi S, et al. Impact of clinical presentation on ischaemic and bleeding outcomes in patients receiving 6- or 24-month duration of dual-antiplatelet therapy after stent implantation: a pre-specified analysis from the PRODIGY (Prolonging Dual-Antiplatelet Treatment After Grading Stent-Induced Intimal Hyperplasia) trial. Eur Heart J 2015;36:1242-51.

67. Bonaca MP, Bhatt DL, Cohen M, et al. Long-term use of ticagrelor in patients with prior myocardial infarction. N Engl $\mathrm{J}$ Med 2015;372:1791-800.

68. Ko DT, Yun L, Wijeysundera HC, et al. Incidence, predictors, and prognostic implications of hospitalization for late bleeding after percutaneous coronary intervention for patients older than 65 years. Circ Cardiovasc Interv 2010;3:140-7.

69. Kazi DS, Leong TK, Chang TI, et al. Association of spontaneous bleeding and myocardial infarction with long-term mortality after percutaneous coronary intervention. J Am Coll Cardiol 2015;65:1411-20.

70. Garay A, Ariza-Solé A, Abu-Assi E, et al. Mid Term Bleeding Risk Prediction After an Acute Coronary Syndrome: An Unsolved Question. Rev Española Cardiol (English Ed 2016;69:527-9.
71. Hamm CW, Bassand J-P. ESC Guidelines for the management of acute coronary syndromes in patients presenting without persistent ST-segment elevation. Jeroen J Bax 2011.

72. Steg PG, James SK, Atar D, et al. ESC Guidelines for the management of acute myocardial infarction in patients presenting with ST-segment elevation. Eur Heart J 2012;33:2569-619.

73. Amsterdam EA, Wenger NK, Brindis RG, et al. AHA/ACC Guideline for the management of patients with Non-ST-Elevation Acute Coronary Syndromes. Circulation 2014:130.

74. Yeh RW, Secemsky EA, Kereiakes DJ, et al. Development and Validation of a Prediction Rule for Benefit and Harm of Dual Antiplatelet Therapy Beyond 1 Year After Percutaneous Coronary Intervention. JAMA 2016;315:1735.

75. Costa F, van Klaveren D, James S, et al. Derivation and validation of the predicting bleeding complications in patients undergoing stent implantation and subsequent dual antiplatelet therapy (PRECISEDAPT) score: a pooled analysis of individual-patient datasets from clinical trials. Lancet 2017;389:1025-34.

76. Raposeiras-Roubín S, Faxén J, Íñiguez-Romo A, et al. Development and external validation of a post-discharge bleeding risk score in patients with acute coronary syndrome: The BleeMACS score. Int $J$ Cardiol 2018;254:10-15

77. Alfredsson J, Neely B, Neely ML, et al. Predicting the risk of bleeding during dual antiplatelet therapy after acute coronary syndromes. Heart 2017;103:1168-76.

78. Serebruany VL, Steinhubl SR, Berger PB, et al. Analysis of risk of bleeding complications after different doses of aspirin in 192,036 patients enrolled in 31 randomized controlled trials. Am J Cardiol 2005:95:1218-22.

79. García Rodríguez LA, Martín-Pérez M, Hennekens $\mathrm{CH}$, et al. Bleeding Risk with Long-Term Low-Dose Aspirin: A Systematic Review of Observational Studies. PLoS One 2016;11:e0160046.

80. Ibanez B, James S, Agewall S, et al. ESC Guidelines for the management of acute myocardial infarction in patients presenting with ST-segment elevation. Eur Heart J. August 2017;2017.

81. Mehran R, Pocock SJ, Nikolsky E, et al. A risk score to predict bleeding in patients with acute coronary syndromes. J Am Coll Cardiol 2010;55:2556-66.

82. Subherwal S, Bach RG, Chen AY, et al. Baseline Risk of Major Bleeding in Non-ST-Segment-Elevation Myocardial Infarction. Circulation 2009:119.

83. Mathews R, Peterson ED, Chen AY, et al. In-hospital major bleeding during ST-elevation and non-ST-elevation myocardial infarction care: derivation and validation of a model from the ACTION Registry ${ }^{\circledR}$ GWTGTM. Am J Cardiol 2011;107:1136-43.

84. Mehta SK, Frutkin AD, Lindsey JB, et al. Bleeding in patients undergoing percutaneous coronary intervention: the development of a clinical risk algorithm from the National Cardiovascular Data Registry. Circ Cardiovasc Interv 2009;2:222-9.

85. Tsai YW, Wen YW, Huang WF, et al. Cardiovascular and gastrointestinal events of three antiplatelet therapies: clopidogrel, clopidogrel plus proton-pump inhibitors, and aspirin plus protonpump inhibitors in patients with previous gastrointestinal bleeding. $J$ Gastroenterol 2011;46:39-45. 